\title{
Index of Myocardial Performance risk assessment by Ultrasound Waves of patients with high blood pressure Nada Abd alkarem(MSc) ${ }^{1}$, Mohamed Ubaid Hussein $(\mathrm{PhD})^{2}$ and Arshad Fouad Redha $(\mathrm{PhD})^{3}$ \\ Abstract
}

Background: It is well known that hypertension results in cardiac overload. This overload is more profound on the left ventricle were systolic high pressure is present resulting in stiff LV.

Objective:To evaluate the index of myocardial performance and stiffness index in patient with hypertension.

Patients and Methods: This study enrolled 105 hypertensive patients and 60 control subjects .Patients with hypertension having an average BP $\geq 140 / 90 \mathrm{mmHg}$. From application of pulse wave echocardiography, the followings parameter are measured: Isovolumetric time of relaxation (IVRT), Isovolumetric time of contraction(IVCT) and time of Ejection (ET) to calculate Myocardial Performance Index (MPI).

Results: The results showed that in hypertensive patients the isovolumetric contraction and relaxation time were increased while the ejection time was decreased, so increasing the index of myocardial performance (IMP) obtained as an indicator for all age groups.

Conclusion: The myocardial performance index increases with increased hardness coefficient of the cardiac ventricular.

Key words: Ultrasound, index of myocardial performance, Blood pressure, Hypertension. Corresponding Author: nadaabdalkrem89@gmail.com

Received: $28^{\text {th }}$ December 2017

Accepted: $28^{\text {th }}$ January 2018

https://doi.org/10.26505/DJM

${ }^{1}$ Department of Physics, College of Science, Anbar University- Anbar - Iraq.
${ }^{2}$ De partment of Physiology and Medical Physics, College of Medicine, Anbar University- Anbar - Iraq.
${ }^{3}$ Department of internal Medical, College of Medicine, Mustansiryah University- Baghdad-Iraq.

\section{Introduction}

Ultrasound is sounds that have frequencies higher than the extent of human hearing frequency, is intended the frequency it is the sound waves produced by the body vibrates per second, and measured frequency by unit called Hertz which equals one cycle (vibration) per second. Most people are able to hear sounds that fall in frequencies between 20 and 20,000 Hz[1].

Although the ultrasound detected before Xrays, for 12 years, but it has many applications in medicine. It is the clinical ultrasound that has a very great importance,

but because the properties of the perception which owned the ultrasound, as well as relatively easy to manage ]2].

The Reflection Technology (ECHO), where the recorded pulse reflected from the border between the two tissues with different acoustic resistance. This technique is based on the principle of work of the sonar. Usually it produces a sound wave by an piezoelectric transducer, strong and weak electrical pulses of ultrasound energy makes the transducer ring at the required frequency ]3]. 
The beam of ultrasound comes from a small device called a transducer. Ultrasound transducers convert electrical to mechanical energy and work on the piezoelectric principle. Piezoelectric materials have the property that if they are mechanically deformed, they produce an electric field that tries to restore the shape. Conversely, if an electric field is applied to a piezoelectric material, in the form of a voltage pulse (typically a few hundred volts), then the material will physically deform [4].

The frequency of ultrasound propagation varies according to the type of tissue, where each tissue has a different propagation velocity than the other. At any given time, the location, size and thickness of the soft tissue in relation to transducer can be calculated. Ultrasound is subject to the laws of optics in relation to refraction, reflection and translation [5].

Ultrasound waves are reflected and absorbed when they meets an interface of differing acoustic impedance. The ultrasonic wave beam is reduced when it is turned off the transducer because of beam reflection, scatter, absorption, and divergence of wave energy at tissue interfaces, the largest ultrasound reflection occurs when the ultrasound beam is perpendicular to the imaged structure, creating a strong reflection or echo [6].

Principles of Doppler Echocardiography: Doppler echocardiography is a method for detecting the direction and velocity of moving blood within the heart, Doppler techniques use ultrasound to evaluate normal and abnormal heart flow and provide quantitative data that is important for clinical decision-making on patients with heart disease [7].

Hypertension:Hypertension is a very diffuse condition where blood is pumped to the artery and leads to many diseases, including heart disease. Blood pressure refers to the result of the first two measures of systolic pressure resulting from left ventricular contraction and the second is diastolic pressure resulting from the relaxation of the left ventricle of the heart [8]. There is a difference in the pressure measurements between the healthy person and the person with high blood pressure where the systolic pressure of people with hypertension> 14 $\mathrm{mmHg}$ and diastolic $>9 \mathrm{mmHg}$. However, lower is better. Young persons may have a blood pressure of $90 / 60 \mathrm{~mm} \mathrm{Hg}$, and as long as that can be maintained, the better one's overall health will be [9]. Hypertension is a silent disease. It is insidious and relentless, the only reliable way to detect hypertension is to regularly check blood pressure [10].

Pulsed Wave Doppler (PW) : Pulse wave Doppler systems use a transducer that alternates transmission and reception of ultrasound in a way similar to the M-mode transducer. One main advantage of pulsed Doppler is its ability to provide Doppler shift data selectively from a small segment along the ultrasound beam, referred to as the "sample volume". The location of the sample volume is operator controlled, an ultrasound pulse is transmitted into the tissues travels for a given time (time $\mathrm{X}$ ) until it is reflected back 
by a moving red cell, it then returns to the transducer over the same time interval but at a shifted frequency [11]. PW Doppler capability is possible in combination with imaging from a mechanical or phased array imaging System[12].

The index of myocardial performance (IMP):By the sum of Isovolumic time of relaxation (IVRT) and Isovolumic time of contraction (IVCT) divided by the left ventricular (LV) time of ejection (ET), the index of myocardial performance (IMP) calculated, The index is considered for patients with dilated cardiomyopathy and post myocardial infarction [13]. The value of this index has a shorter Isovolumic time and an preserved LV ejection time (ET), but was unrelated to heart rate, preload, and mean arterial pressure.

\section{Patients and Methods}

The study included 105 hypertensive patients and 60 control subjects referred to the echocardiography unit at Al-Yarmouk Teaching Hospital/ Baghdad. Patients with hypertension having an average $\mathrm{BP} \geq 140 / 90$ mmHg. The study was carried out as follows:- The population of study was divided into two main groups.

The first main group represents controls group includes 60 normal , 22 males and 38 females. then, the same group was sub divided according to age into three sub groups (20-50),(51-70) and (71-90). The second main group represents patients with hypertension (HT) include 105 patients, 46 males and 59 females, also sub divided into three sub groups (20-50),(51-70) and (71-90).
Before considering patient for study we have excluded all other diseases apart from hypertension. Patients were subjected to a series examination; these were ECG, blood test for diabetes mellitus and echocardiography to exclude valvular disease and / or congenital anomaly. Patients' consents were taken for using their data in the present study. The height and weight were measured for both patients and normal subjects. The history of HT was recorded to help the cardiologist for better evaluation of echocardiographic diagnosis.

To reduce percentage of error in the IVRT and IVCT calculations, total systolic time was taken and subtracted from ET value. The stiffness index was calculated by the equation [(E/Ea)/LVIDd] [14].

\section{Statistical Analysis}

The statistical analyses were carried out by the using excel program. For all values were calculated mean values with \pm standard deviations, percentage change for every parameter for control and patients groups were calculated and a test for significance between the mean values for each of the two groups was carried out by unpaired student $t$ test, $\mathrm{p}$ value $\quad 0.05$ was considered significant.

Echocardiography System Used in This Work: M-mode, two-dimensional (2-D) and pulse wave Doppler echocardiography examinations were obtained by using a commercial instrument with 2-5 $\mathrm{MHz} \mathrm{S} 3$ transducer, vivid E9 GE echocardiographic device made in USA, supplied by Medicine Company Fig. (1). The system is capable to 
carry out a variety of applications so that it is departments, including general abdomen, optimized for use in a variety of ultrasound vascular, cardiac, breast and etc.

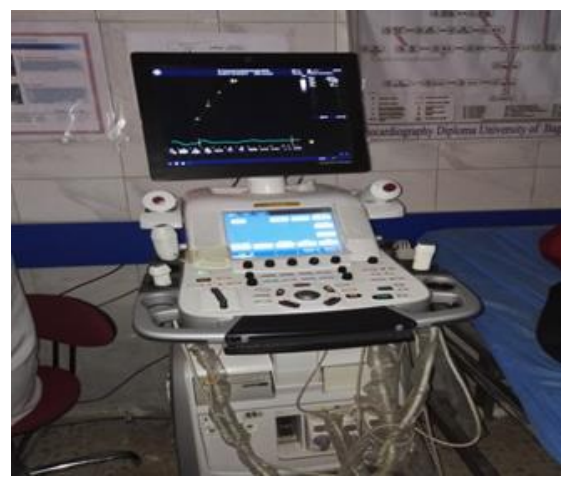

Figure (1): The Ultrasonography Machine.

The system offers many diagnostic possibilities such as B-Mode, M-Mode and Spectral Doppler (pulsed and continuous wave).

\section{Results}

Because the myocardial stiffness and the stiffness index are influenced by aging, we divide the study population into three categories each category involves one age group. The first category is for age group ranging from (20-50 yr.), the second is for age range (51-70yr.) and the third is for age range (71-90yr.).

\section{Anthropometry}

The table (1) represents the values of age, height and weight for patients and control groups. The differences in the average values of height between patients and control groups were insignificant ( $p$ value more than 0.05 for the all sub age groups, while the weight was significant ( $\mathrm{p}$ value $(0.008)$ and (0.0001) respectively for age ranges (20-50), (51-70), while for age range (71-90) was insignificant ( $p$ value 0.621 ). 
Index of Myocardial Performance risk assessment by Ultrasound Waves of patients with high blood pressure Nada Abd Alkarem

Table (1): Features for study population.

\begin{tabular}{|c|c|c|c|c|}
\hline \multirow{2}{*}{ Parameter } & control & HT & Change\% & \multirow{2}{*}{ p-value } \\
\hline & Mean \pm SD & Mean \pm SD & $(\mathrm{HT}-\mathrm{C}) / \mathrm{C} \times 100$ & \\
\hline \multicolumn{5}{|c|}{ the range of age $(20-50)$ years } \\
\hline Age & $41.93 \pm 15.39$ & $57.84 \pm 13.63$ & 37.94 & 0.648 \\
\hline Height $(\mathrm{cm})$ & $165.40 \pm 9.58$ & $168.04 \pm 9.54$ & 1.60 & 0.499 \\
\hline weight $(\mathrm{kg})$ & $74.00 \pm 12.65$ & $83.71 \pm 13.80$ & 13.12 & $0.008 *$ \\
\hline \multicolumn{5}{|c|}{ the range of age $(51-70)$ years } \\
\hline Age & $58.28 \pm 15.73$ & $58.15 \pm 13.61$ & 36.60 & 0.147 \\
\hline Height $(\mathrm{cm})$ & $165.20 \pm 9.75$ & $168.04 \pm 9.53$ & 1.72 & 0.062 \\
\hline weight $(\mathrm{kg})$ & $74.03 \pm 12.51$ & $83.56 \pm 13.87$ & 12.87 & $0.0001 *$ \\
\hline \multicolumn{5}{|c|}{ the range of age $(71-90)$ years } \\
\hline Age & $76.4 \pm 17.52$ & $58.23 \pm 13.67$ & 32.34 & 0.657 \\
\hline Height $(\mathrm{cm})$ & $163.84 \pm 9.56$ & $168.18 \pm 9.52$ & 2.65 & 0.349 \\
\hline weight $(\mathrm{kg})$ & $73.07 \pm 13.02$ & $83.58 \pm 13.86$ & 14.38 & 0.621 \\
\hline
\end{tabular}

*HT=hypertension, $\mathrm{C}=$ control 
Index of Myocardial Performance risk assessment by Ultrasound Waves of patients with high blood pressure Nada Abd Alkarem

Total Systolic Time(TST), Ejection value was insignificant ( $\mathrm{p}$ value $(0.500)$, Time(ET) and Index of Myocardial (0.203) and (0.464) respectively for the three Performance(IMP): The difference in IMP age groups ), as illustrated in table (2).

Table (2): Doppler echocardiography determining TST, ET, and IMP.

\begin{tabular}{|c|c|c|c|c|}
\hline \multirow{2}{*}{ Parameter } & control & HT & Change\% & \multirow{2}{*}{ p-value } \\
\hline & Mean \pm SD & Mean \pm SD & $(\mathrm{HT}-\mathrm{C}) / \mathrm{C} \times 100$ & \\
\hline \multicolumn{5}{|c|}{ the range of age $(20-50)$ years } \\
\hline $\mathrm{TST}(\mathrm{ms})$ & $373 \pm 26.93$ & $438.79 \pm 47.86$ & 17.64 & $0.0001 *$ \\
\hline $\mathrm{Et}(\mathrm{ms})$ & $245.95 \pm 36.85$ & $279.93 \pm 59.34$ & 13.82 & $0.006^{*}$ \\
\hline MPI & $0.51 \pm 0.20$ & $0.58 \pm 0.20$ & 13.73 & 0.500 \\
\hline \multicolumn{5}{|c|}{ The range of age $(51-70)$ years } \\
\hline $\mathrm{TST}(\mathrm{ms})$ & $374.39 \pm 26.70$ & $439.32 \pm 48.09$ & 17.34 & $0.0001 *$ \\
\hline $\mathrm{Et}(\mathrm{ms})$ & $245.93 \pm 37.44$ & $280.92 \pm 59.18$ & 14.23 & $0.041 *$ \\
\hline MPI & $0.52 \pm 0.20$ & $0.56 \pm 58.18$ & 7.69 & 0.203 \\
\hline \multicolumn{5}{|c|}{ the range of age $(71-90)$ years } \\
\hline $\mathrm{TST}(\mathrm{ms})$ & $377.89 \pm 21.92$ & $439.53 \pm 48.20$ & 16.31 & $0.0001 *$ \\
\hline $\mathrm{Et}(\mathrm{ms})$ & $241.42 \pm 37.83$ & $280.48 \pm 59.12$ & 16.18 & $0.034 *$ \\
\hline MPI & $0.56 \pm 0.20$ & $0.57 \pm 0.23$ & 1.79 & 0.464 \\
\hline
\end{tabular}

*TST= Total Systolic time, ET=Ejection time 


\section{Effect of IMP With Stiffness Index:}

For the all age groups there is a direct relationship between Myocardial Performance Index (IMP) and Stiffness
Index (ST). As the stiffness index increases, the myocardial performance index increases also, as shown in figure (2), (3) and (4).

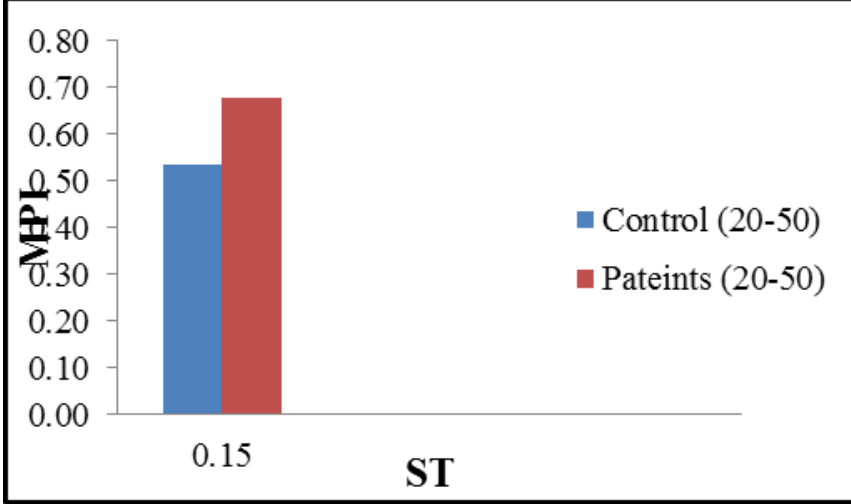

Figure (2): Relationship between ST and IMP, age between(20-50years).

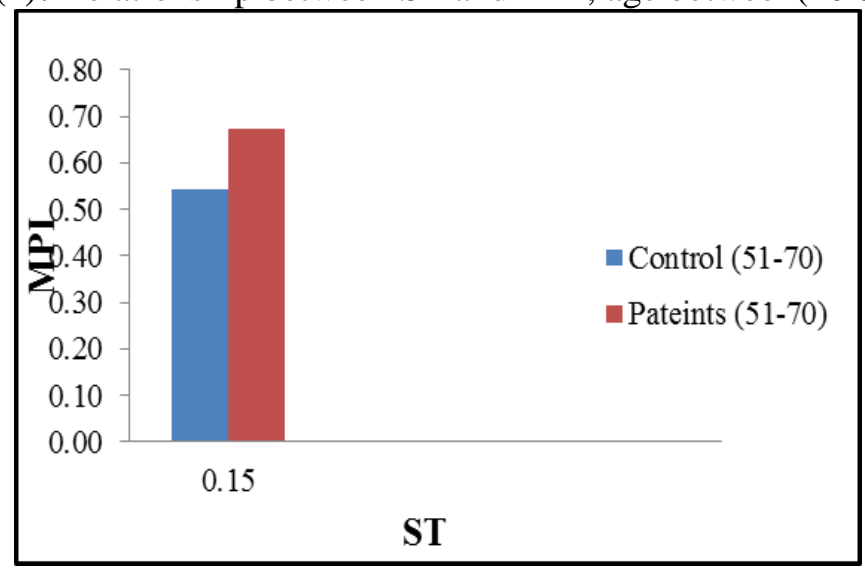

Figure (3): relationship between ST and IMP, age between(51-70years).

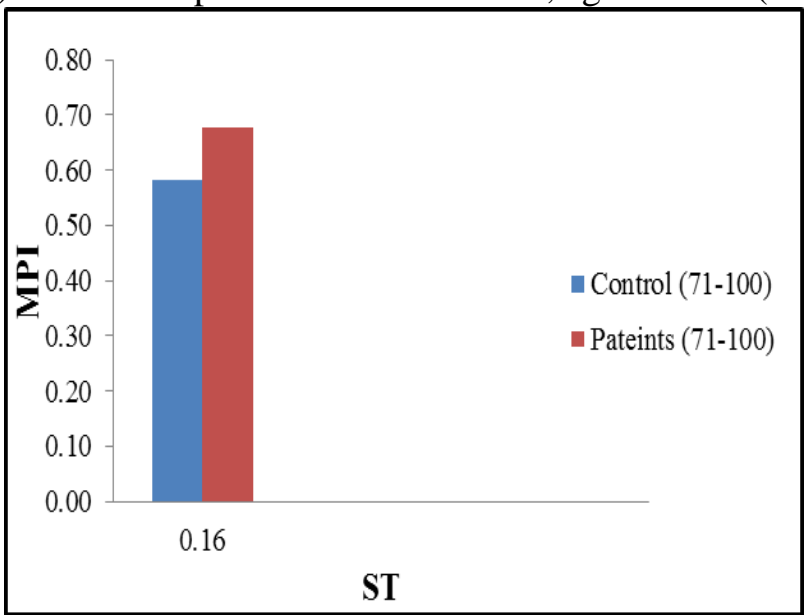

Figure (4): relationship between ST and IMP, age between (71-90years). 


\section{Discussion}

The delay of valve opening due to extended relaxation causes increased IVRT, that is the index of the rate of relaxation of the heart muscle. Several factors affect on time of relaxation, aging and weakness of relaxation, as well as changes in heart rate and systolic function[15].

For most patients who have diastolic dysfunction, the initial or earliest abnormality is termed impaired relaxation. This results from the loss of elastic recoil of the left ventricle in early diastole leading to a reduction in the force by which blood is sucked through the mitral valve. Hemodynamiclly, this lead to a delay or prolongation of the left ventricular pressure curve during isovolumic relaxation. This prolongation, in turn, causes a delay in mitral valve opening and a prolongation of the isovolumic relaxation time (IVRT) as seen in table (2).

Increasing IMP is an index for systolic and diastolic dysfunction, these results are in agreement with the previous study of $\mathrm{F}$. Cacciapuoti et al., (2007) [16].

In Fig. (2) and (3) the increase in patients and controls diagrams for two age groups (20-50yr) and (51-70yr) index a gradual deterioration in the performance of the heart muscle when the coefficient of stiffness is increase . at the old age (71-90) the diagram of control was highest increase in the heart muscle index was recorded with the increase of stiffness index, which indicates a rapid decline in the age of the advanced as shown in Figure (4).
This study shows that the value of MPI was insignificant change between patients and controls, that's means there is a slight difference between them. This result agreement with Zainab A. Al-Rikabi et. al. (2017) [17] which got the same result but with significant $\mathrm{p}$ value.

\section{Conclusion}

When the times of relaxation and contraction increases with ET decrease, the myocardial performance index will increase with the increase of the ventricular hardness coefficient.

\section{Acknowledgements}

We would like to express special words of thanks with deepest appreciation of the echocardiography unit at Al-Yarmouk Teaching Hospital/ Baghdad.

\section{References}

[1] John R. Cameron ,James G. Skofronic ," Medical Physics", published simultaneously in Canada ,p265-275, 1994.

[2] A.E. Abbas , F.D. Fortuin , N.B. Schiller , C.P. Appleton, C.A. Moreno, S.J. Lester, J Am Coll Cardiol., 19,41 (2003)1021-7.

[3] D.A. Lichtenstein, "Basic notions in critical ultrasound". In: D.A. Lichtenstein, editor. Whole body ultrasonongraphy in the critically ill New York: Springer, (2010) 310.

[4] W. H. Gaasch, O. H. L. Bing and I. Mirsky , Eur Heart J, 3 (1982) 139-145.

[5] H.C. Dokainish, Curr. Opin. Cardiol., 19, 5 (2004) 437-41. 
[6] P. M. Srivastava, L. M. Burrell, P. Calafiore, Eur J Echocardiography, 6(2005) 97-106.

[7] S.I. Xiao-dong, L.I.U. Zhi-yue, Wang Jin-rui , J. Y Yang, Xu Xia, Journal of Medical Biomechanics, 02(2006) 125-128.

[8] A. C. Guyton, J. E. Hall "Textbook of Medical Physiology".11thed. Philadelphia: WB Saunders Company,(2006) 110-114.

[9] Y. Kokubo, K. Kamide, Circ J., 73(2009) 1381-1385.

[10] F.M. Sacks, H. Campos, N Engl J Med., 362(2010) 2102-2112.

[11] P. J. Fish, C. R. Hill, "Physical Principles of Medical Ultrasonics", Ellis Horwood Ltd, (1986) 338 -376.

[12] D.G. Julian, J.C. Cowan, J.M. Mclenachan, "Cardiology", Seventh edition,
Edinburgh W.B. Saunders Company, (1998) 69-74.

[13] Sh. H. Hayes, Mayo clinic, (1999) 4, 5, 36, 37.

[14] K. Abd El Salam, Heart Mirror Journal, 4, 2 (2010) 186-191.

[15] M. K. Seok , W.H. Jong., R. Se-Joong, and C. Namsik, Yonsei Medical Journal, 39 ,5 (1998) 446-452.

[16] F. Cacciapuoti, E. Manfredi, R. Marfella, F. Cacciapuoti, G. Caruso, G. Nittolo. Heart Views, 8, 2(2007) 34-39. [17] Zainab A. Al-Rikabi, Ghassan T. Saeed and Ghazi F. Haji, Journal Fac Med Baghdad, 59,1 (2017) 65-70. 